\title{
L'Image du protestant et le conseil municipal d'Aix au XVIe s.*
}

\author{
CLAIRE DOLAN
}

Depuis quelques années, les historiens recherchent au-delà des événements l'expression d'attitudes directement liées aux valeurs culturelles. ${ }^{1}$ La période des guerres de religion n'a pas échappé à cette lecture nouvelle et les travaux de Janine Estèbe, comme ceux de Natalie Davis, ont rapidement placé cette période au coeur d'une interprétation dynamique de la violence au XVIe siècle. ${ }^{2}$ D'autre part, l'association a été faite entre les systèmes de valeurs et les relations qu'ils entraînaient au sein de la société et l'on a vu dans les conflits religieux du XVIe siècle une volonté des notables "d'imposer aux classes inférieures une religion plus prégnante, une morale plus sévère, un contrôle plus strict des gestes de la vie quotidienne." 3

Les travaux récents se sont volontairement placés dans la perspective des luttes, des conflits, de la violence issus de la cohabitation confessionnelle. Bien que cette vision soit tout à fait pertinente dans la mesure où elle s'attache à expliquer les comportements par les comportements euxmêmes, je souhaiterais quant à moi situer ma réflexion au niveau de la représentation que se font les deux groupes l'un de l'autre, en dehors de l'événement conflictuel même. Ce choix s'est imposé par la qualité des sources qui ne permettait pas d'étudier de façon critique la violence ellemême, dans la mesure où ces renseignements étaient toujours donnés par des sources de seconde main qui permettent plus de cerner les positions du rédacteur que celles des acteurs de l'événement. ${ }^{4}$

Dans cette perspective, la rigueur eût exigé que les deux partis aient la parole. A Aix-en-Provence cependant, au XVIe siècle, les catholiques parlent plus fort que les protestants ou du moins ils y ont tribune. C'est donc leur représentation des protestants que j'ai retenue, représentation qui s'exprime pour le cas présent entre les années 1559 et 1568.

Une série de problèmes théoriques se posent au départ. Avec les pro-

* Communication donnée le 23 mai 1979 lors d'une séance de travail de la société Canadienne d'Etudes de la Renaissance tenue a l'University of Saskatchewan, Saskatoon, Saskatchewan. 
testants, c'est un groupe culturel différent du groupe culturel dominant qui se met en place, ce qui pose le problème de la représentation en termes de relations inter-culturelles, en termes d'acculturation. Les relations inter-culturelles entraînent une évaluation de la culture de chacun par l'intermédiaire entre autres de l'ethnocentrisme qui suppose la supériorité de ses propres valeurs sur celles des adeptes d'un système culturel autre. ${ }^{5}$ Dans quelle mesure les conflits entre catholiques et protestants au XVIe siècle reflètent-ils les réactions aux "transmissions culturelles" qui s'effectuent entre "sociétés de cohérences culturelles dissemblables"?6 Problème fondamental que le présent exposé n'a pas la prétention de résoudre. C'est dans le cadre de cette interrogation globale cependant qu'il faut placer la présente recherche. Il s'agit plus précisément de voir si l'image que se font les catholiques des protestants résulte de l'observation d'une culture considérée comme fondamentalement différente, étrangère ou si elle est davantage associée à l'idée d'une différence momentanée qui n'altère pas le fond culturel commun.

\section{Les sources et la méthode}

Pour retrouver les éléments qui permettent de reconstituer cette image en tenant compte des différents facteurs qui en façonnent l'aspect, il faut utiliser des sources qui livrent la pensée des acteurs tout en permettant d'identifier ces derniers nommément et socialement. Les registres de délibérations municipales paraissent sur ce point particulièrement riches.

D’une part, ils révèlent la réaction périodique d'une partie des notables aux événements qui affectent les citadins; ils précisent qui sont les membres du conseil qui assistent aux délibérations, indiquent quels sont ceux qui font les propositions et, quand les minutes ont survécu aux extensoires, comment chaque membre a voté. Il est donc possible de retrouver les prises de position des notables de la ville quitte à préciser grâce à d'autres sources (les archives notariales surtout) l'activité économique et les liens sociaux de ces individus.

D'autre part, ces registres constituent un corpus suffisamment homogène pour permettre d'être traités de façon sérielle à partir d'une grille qui dépasse le niveau thématique. Des précautions préliminaires s'imposent toutefois si l'on veut retrouver dans le langage des délibérations une image exacte des protestants. Les méthodes linguistiques que l'histoire a découvertes ces dernières années ${ }^{7}$ doivent être utilisées, dans le cas présent, avec beaucoup de circonspection. Le caractère même du document et la variation de l'orthographe ont fait mettre de côté une analyse automatique du texte. Les calculs de fréquence par rapport à l'ensemble des mots du texte sont évidemment absents. Bien que notre démarche 
ait voulu s'écarter d'une lecture thématique du texte, c'est malgré tout un thème qui a orienté notre étude. La problématique retenue, plus que le texte lui-même, a suggéré la voie à suivre. ${ }^{8}$ Il ne s'agit donc pas ici de tenter d'analyser un discours mais bien de voir comment s'organise ce discours face à un thème donné, préalablement choisi par l'historien.

Deux notions étaient à considérer au départ: celle d'étranger, celle de protestant. Une étude conduite parallèlement sur les deux notions aurait donné plus de crédibilité aux résultats. Il s'est avéré toutefois que la fréquence du terme étranger était insuffisante pour relever d'une telle étude et que la notion elle-même était trop vague pour justifier d'un réseau de synonymes. Le problème était différent pour la notion de protestant puisque, si ce terme même n'est jamais utilisé, ses synonymes sont faciles à identifier et ils apparaissent fréquemment dans le texte. Nous avons donc recherché dans les délibérations municipales tous les synonymes du mot protestant et étudié les réseaux sémantiques dans lesquels ils se situaient par l'intermédiaire des catégories suivantes:

10 - action de (c'est-à-dire toute action faite par le protestant)

20 - action sur (c'est-à-dire toute action subie par le protestant)

30 - opposition (ce à quoi le rédacteur oppose le protestant, par l'intermédiaire d'une préposition (contre, etc.) ou de façon implicite)

40 - association (c'est-à-dire ce à quoi le protestant est associé)

50 - qualification (comment on qualifie le protestant, ainsi que les noms qu'on lui donne). ${ }^{9}$

Les résultats ont ensuite été traduits de manière à effectuer des regroupements qui permettent l'interprétation.

A qui cette image du protestant peut-elle être attribuée? J'ai déjà manifesté mon inquiétude concernant l'intervention du rédacteur dans la narration qu'il fait des intentions des acteurs. ${ }^{10}$ Les délibérations municipales nous sont livrées par l'intermédiaire d'un greffier, notaire de la ville qui inscrit pour le conseil les résultats de la réunion. Les termes utilisés et sur lesquels repose toute notre étude révèlent-ils davantage l'image que se fait le notaire du protestant ou l'image que s'en font les conseillers, laquelle est alors finalement transcrite? Le problème serait sans issue si nous n'avions la possibilité de comparer dans quelques cas, les minutes des délibérations et la version finale qui en est restée. Il est fort probable que les minutes, écrites rapidement, sont assez fidèles aux expressions et au vocabulaire entendus par le notaire quand il transcrit. Des corrections pourraient par contre intervenir au moment de la rédaction finale alors que le notaire prend la peine de compléter les titres de chacun et de préciser les transitions absentes dans la minute. La comparaison des minutes et du texte final permet de constater que les textes courts sont semblables dans un cas comme dans l'autre. Les textes plus longs, s'il arrive qu'ils soient structurés différemment, ne présentent pas de variation de vocabulaire. ${ }^{11}$ Les discours par contre qui sont fort rares 
doivent être traités avec grande prudence. Ainsi le discours de sortie de charge du seigneur de Pourrières diffère considérablement d'une version à l'autre. ${ }^{12}$ Dans quelle mesure le notaire a-t-il tenté de restituer le discours du consul qu'il avait d'abord abrégé? Il est difficile de le percevoir et l'utilisation des discours devrait être assujettie à un appareil critique fort sevère. Heureusement, la notion de protestant n'a pas été soumise à ce type de discours. On peut dès lors être à peu près assuré que la représentation véhiculée par le texte est celle de l'orateur.

\section{Le conseil municipal et ses orateurs}

Le conseil municipal détenait le pouvoir à Aix. Toutes les décisions importantes lui étaient soumises et elles ne devenaient effectives qu'après son approbation. Les conseillers étaient cooptés en même temps que les consuls qui étaient quant à eux les représentants du conseil pour les affaires courantes. Durant la période étudiée, le premier consul nomme les 3 consuls et l'assesseur de l'année suivante mais cette nomination doit être approuvée par les conseillers qui votent sur chaque poste au moyen d'une fève noire ou blanche. ${ }^{13}$ Le consul doit présenter des noms au conseil tant que celui-ci ne se montre pas d'accord avec son choix. Le premier consul est toujours recruté dans la noblesse fieffée, le deuxième consul parmi les gentilshommes et le troisième est issu de la bourgeoisie. ${ }^{14}$ L'assesseur qui suit le premier consul en dignité est choisi parmi les avocats d'Aix.

Quand il est présent, c'est toajours le premier consul qui suggère au conseil les points à traiter. Il est rare que des conseillers amorcent euxmêmes ce débat. Bien qu'ils participent aux discussions, ils ont une faible part à l'orientation de celles-ci. Consuls et assesseur suggèrent les mesures à prendre, les conseillers acceptent ou refusent mais les délibérations municipales reflèteront donc d'abord la représentation des consuls et de l'assesseur et périodiquement celle des hauts personnages qui ont fait convoquer le conseil (gouverneur, lieutenant du roi, etc.).

L'étude d'un corpus plus important aurait pu nous permettre de séparer la représentation véhiculée par le corps de ville de celle des autorités provinciales. Dans la mesure où ces visites donnent plus ou moins lieu à un discours sur les protestants et où ce discours ne tranche pas avec celui des autorités municipales, nous avons confondu ces représentations.

\section{LES RESULTATS}

\section{Le contexte de production}

Il serait long et ardu de restituer les événements qui donnent à la période étudiée ses dimensions réelles. Il ne s'agit pas de raconter ici les circonstances que les historiens du temps ont narrées avec une certaine délecta- 
tion mais simplement de resituer les documents étudiés dans leur contexte de production. Bien que l'année 1559 soit considérée comme le moment où le protestantisme atteint sa plus grande expansion en France, ${ }^{15}$ il faut attendre 1560 pour que la communauté aixoise réagisse collectivement à ce phénomène. La tentative de Mauvans et de ses soldats protestants $\mathrm{du}$ Luberon pour prendre la ville par surprise, avec la collaboration de huguenots aixois, devait échouer ${ }^{16}$ mais laisser aux Aixois un souvenir vivace. Le ler novembre 1561 , le conseil d'Aix élit à sa tête Ulmo, Nas et Taurel qui devaient être démis de leur poste, au cours de l'année, par le comte de Tende, gouverneur de Provence, pour leur insoumission et leur fanatisme. Elu en même temps qu'eux, le premier consul Durand de Pontevès, seigneur de Flassans s'est surtout fait remarquer quant à lui par "son enthousiasme farouche, sa dureté, sa violence et son fanatisme, que ne tempéraient ni une intelligence cultivée, ni les sentiments tendres de la famille". ${ }^{17}$ Chef de bande catholique, on lui attribue pour cette période la terreur qui régnait autour d'Aix. Ses activités parallèles obligeront Flassans à un certain absentéisme des réunions du conseil. On notera cependant que sa présence donne toujours lieu à des prises de position à l'égard des protestants, prises de position que le conseil ne manque pas d'ailleurs de ratifier. Je n'ai pas isolé ici l'image fanatique esquissée par Flassans en 1561-62 de l'image globale constituée sur dix années. Dans la mesure où cet excès même contribue à tracer cette image, il a fallu le laisser participer à l'interrogatoire. Flassans n'étant contredit par personne à l'intérieur du conseil, il faut présumer qu'il emportait l'assentiment de ses collègues dans sa façon de traduire la réalité. En cette année 1562, Aix est le théâtre de sanglantes émeutes où les catholiques jouent le rôle qu'ils reprocheront bientôt aux protestants. Le fanatisme semble donc dépasser la personnalité des consuls et de l'assesseur.

Des nombreux édits qui fleurissent en cette période, c'est l'édit de 1563 qui eut le plus de retentissement au conseil de ville. Il accordait aux protestants la liberté de conscience et leur rendait la propriété de leurs biens mais Aix n'était pas parmi les villes où ils pouvaient exercer publiquement leur culte. ${ }^{18}$ Trève difficile à expliquer et qui devait être de courte durée, elle marque cependant une étape dans l'agitation. La visite de la reine mère et du roi à Aix en 1564 entraîne une relative tranquillité. ${ }^{19}$

Les délibérations semblent peu sensibles aux protestants après 1564 . Les mentions sont rares et l'on peut s'interroger sur les raisons de ce silence. ${ }^{20}$ Pas un mot, entre autres, de la défection de l'archevêque d'Aix, Jean de St-Chamond, qui déchira sa mitre en chaire le 25 décembre 1566 et passa au protestantisme! ${ }^{21}$ Les actions individuelles qui n'avaient pas d'incidence immédiate sur la sécurité de la ville ou sur ses finances ne jouissent d'aucune attention au conseil. De 1565 à 1568, les combats 
continuent en Provence mais Aix n'est pas menacée et l'on ne retrouve plus en son sein les violences qui l'avaient divisée de 1560 à 1563.

\section{La communauté protestante aixoise}

On sait peu de choses de la communauté protestante aixoise si ce n'est par des sources de seconde main. Quelques indices nous sont cependant donnés qui accordent à la période 1560-1562 une importance fondamentale dans le développement de la communauté. Le 4 avril 1569, l'official Honoré Moreti reçoit une dizaine d'abjurations. La plupart des transfuges expliquent qu'ils ont adopté la nouvelle religion entre les années 60 et 63 , par curiosité ou par incitation et que leurs complices sont en "sy grand nombre qu'il ne les sauroiet nommer." 22

L'état présent des recherches ne permet guère de préciser les catégories sociales principalement touchées: avocats, procureurs au Parlement, bourgeois, médecins, artisans, marchands, conseillers au Parlement comptent parmi les protestants mais une statistique un tant soit peu vraisemblable est encore impossible à mettre en oeuvre. ${ }^{23}$

On comprendra dès lors qu'il ne s'agisse pas ici de vérifier une hypothèse portant sur les conflits sociaux sous-jacents aux conflits religieux. . . ${ }^{24}$

\section{L'image du protestant au conseil municipal d'Aix}

Les noms donnés par les catholiques aux protestants sont révélateurs de l'influence subie par les Aixois au niveau du vocabulaire. En effet, ils ne suivent ici que la tendance qui a cours dans tout le royaume et indiquent surtout, par l'adoption du vocabulaire national, leur adhésion au mouvement catholique français. ${ }^{24}$ C'est donc par rapport à l'ensemble du royaume que l'on devrait étudier cette évolution.

Pour la période considérée, il est clair que la désignation des protestants par un terme propre, dépourvu de jugement moral, est ce qu'il y a de plus fréquent. Ce sont des luthériens (6 mentions), des huguenots (4) mais ce sont surtout gens "de la nouvelle religion" (14) ou plus tard ceux "de la religion" tout simplement (7).

Les qualifications qu'on leur attribue et qui sont souvent devenues expressions consacrées sont plus intéressantes. On pourrait les classer en 6 catégories différentes (tableau 1).

L'ensemble des catégories permet déjà d'entrevoir que les protestants ne sont pas situés prioritairement par rapport à leur appartenance à la communauté aixoise. Le tort qu'ils font à la religion mais presque tout autant le désordre public qu'ils créent prennent le pas sur cette qualité d'appartenance. D'ailleurs, sait-on bien qui sont ces protestants qui perturbent l'ordre public et s'écartent de la religion chrétienne? On sait qu'ils sont nombreux, l'individu protestant n'apparaît qu'exceptionnellement 
Tableau 1

\begin{tabular}{|c|c|c|}
\hline \multicolumn{3}{|c|}{ QUALIFICATIONS } \\
\hline $10-$ & Les indéfinis (on, aulcung, manière de gens, etc) & 11 mentions \\
\hline 20- & Les quantificateurs ( $p l$ usieurs, grand nombre, etco) & 7 mentions \\
\hline 30- & $\begin{array}{l}\text { Les qualificatifs à connotation morale (mauvais, } \\
\text { malins, méchants) }\end{array}$ & 6 mentions \\
\hline $40-$ & $\begin{array}{l}\text { Ceux qui expriment un rapport avec la religion chré- } \\
\text { tienne (mal sentant de la foi, mal affectionné à } \\
\text { la religion, etc.) }\end{array}$ & 12 mentions \\
\hline $50-$ & $\begin{array}{l}\text { Ceux qui expriment un rapport avec l'ordre public } \\
\text { (séditieux, rebelles, perturbateurs, etco) }\end{array}$ & 11 mentions \\
\hline $60-$ & $\begin{array}{l}\text { Ceux qui expriment un rapport avec la ville } \\
\text { (de la ville, étranger, etc.) }\end{array}$ & 7 mentions \\
\hline & TOTAL: & 54 mentions \\
\hline
\end{tabular}

et toujours pour mettre en évidence le grand nombre de ceux qui le suivent. Cependant, les protestants apparaissent aux Aixois comme un groupe qui demeure dans ses éléments largement indéfini dans la mesure où l'on s'intéresse d'abord aux résultats de l'action de ce groupe vis-à-vis des deux piliers de la société, l'ordre public et la religion. Il est intéressant de noter ici que l'utilisation de l'indéfini introduit toujours un effet d'ambiguité et permet à celui qui l'emploie d'éviter de se justifier.

Le réseau des oppositions vient préciser les données précédentes puisque les catégories peuvent être un peu mieux définies (tableau 2).

Il est remarquable que les protestants soient ici opposés à des êtres inanimés dans la plupart des cas. Les Aixois voient davantage les protestants dans une relation avec les institutions qui sont les bases de leur société qu'avec des individus. Il semble clair que leur opposition à ce qui pourrait grossièrement être appelé le système culturel aixois contribue largement à définir les protestants. Pouvons-nous aller jusqu'à hiérarchiser cette opposition? Les valeurs fondamentales sont ici encore largement ébranlées. Les protestants ont le tort de s'opposer au roi et à son autorité, la fréquence de l'accusation en fait foi. Notons toutefois que les consuls portent là une accusation qui pourrait leur être retournée allègrement. Ne soyons pas trop désobligeant cependant. Jamais, dans les délibérations du conseil, une opposition au roi ou à ses édits n'a été exprimée comme telle. Quand le conseil contestait l'application d'un édit, il se prévalait toujours du respect d'un autre édit ou invoquait la mauvaise 


\section{OPPOSITIONS}

CATEGORIES

NOMBRE DE MENTIONS

10- Morale(devoir, raison, bons, etc.)

20- Religion

30- Repos et sûreté publics(tranquillité, etc.)

40- Autorité du roi (obēissance, édits du roi, etc.) 24

5o- Ville (ville, députés du conseil, consuls)

6o- Peuple(peuple, gens qui travaillent)

70- Autres (garde, cour)

TOTAL:

interprétation de l'édit en question. Jamais il ne s'est opposé ouvertement au roi. L'accusation prend donc encore plus de poids. ${ }^{26}$ Opposition au roi d'abord, opposition à la religion ensuite, ce qui n'étonne guère. La troisième catégorie en importance vient renforcer notre interprétation. Si les valeurs fondamentales sont atteintes, tout l'équilibre du système culturel est touché, ce que signifie bien l'importance du repos et de la tranquillité publics dans le réseau des oppositions. L'opposition des protestants aux valeurs du conseil aixois remet en question toute la vie communautaire. Perturbation temporaire? Les résultats jusqu'à présent nous laissent perplexe.

Plus nombreuses, les associations nous permettront peut-être d'entrevoir plus clairement cette perturbation (tableau 3).

Les protestants sont d'abord associés à la violence. La négation de l'autorité en place prend ici une forme active. Tant par le vocabulaire militaire que par les perturbations violentes auxquels elle est associée, elle exprime la mise en danger de toute la sociéte. Cette violence dépasse largement la religion ou les individus, elle touche directement les fondements du système. Là où la violence est absente, c'est le groupe qui demeure malgré tout prioritaire dans l'image qu'on se fait du protestant. Celui-ci ne peut guère être considéré qu'en assemblée et l'individu auquel on l'associe parfois est aussi défini par le groupe. L'étranger, le vagabond ou le protestant s'écartent comme groupe des normes admises.

Il faut cependant noter que cette précision donnée de l'image du protestant se constitue par l'inclusion du protestant à un groupe auquel il participe mais qui ne le définit pas directement. Ainsi les protestants sont associés aux ennemis, aux vagabonds, etc. Ils ne sont pas ouverte- 
Tableau 3

\section{ASSOCIATIONS}

\section{NOMBRE DE MENTIONS}

10- Associations à des êtres animés sans con-

notation violente

A- Individus (étrangers, vagabonds, suspects, etcd 12 dont 3 à connotation positive

B- Groupes (diète, assemblées, etc.)

20- Association à des inanimés sans connotation violente

- Religion (exercice, prêche, Genève)

30- Association à des formes de violence

A- Violence contre individus (meurtres, vols, etc) 7

B- Violence contre religion (sacrilèges, brisement de croix, etc.)

C- Perturbation violente présumée (danger, hostilité, péril, etc.)

D- Perturbation violente(troubles, émotions, séditions, etc.)

E- Aspect militaire (adversaires, invasions, port d'armes, etc.)

40- Association à l'autorité du roi

- Désobéissance, rebelles du roi, etc. dont 2 mentions à connotation positive

50- Autres

- Dommages, excès, scandale, inconvénient, insulte

TOTAL:

ment déclarés ennemis et vagabonds. De même sont-ils associés aux émotions et aux troubles sans nécessairement en porter toute la responsabilité. Verrait-on apparaître ici la notion de bouc émissaire qui a tenté déjà les historiens de la violence? L'association est trompeuse dans la mesure où elle permet mal de cerner l'image du protestant et de la dégager des conséquences que les Aixois tirent de cette image. Il vaut mieux revenir aux protestants eux-mêmes et voir comment le réseau des actions qu'on leur prête peut compléter cette image (tableau 4). 


\section{ACTIONS DE}

CATEGORIES

NOMBRE DE MENTIONS

10- Action liée à la religion (prêcher, faire exercice, etc.)

20- Action à caractère passif (être à tel endroit, pouvoir, savoir, vivre, etc.)

30- Action liée à la volonté d'agir (vouloir, menacer, chercher, désirer, etc.)

40- Action préparant une offensive (venir, entrer, survenir, etc.)

50- Action offensive(prendre armes, saccager, etc.)

60- Action manifestant un recul (sortir, se retirer, quitter armes)

70- Action sans connotation (mettre, écrire, descendre, passer, etc.)

80- Autres (commettre)

TOTAL:

La violence qui avait caractérisé le réseau des associations est ici mise en lumière et précisée. Les protestants exercent une action offensive et c'est là leur principale caractéristique. Par rapport aux gestes qu'ils posent, la religion est loin de susciter la plus grande inquiétude. Leur action comme groupe est agressive et c'est comme agresseurs qu'ils sont perçus. Cette agression par contre est d'autant plus marquée qu'elle n'a pas à être réalisée. Elle existe en puissance et cette constatation même pousse le protestant plus loin encore dans son rôle de perturbateur. Des 155 mentions d'actions faites par les protestants, 40 portent sur des intentions qu'on leur prête. Ils "menacent de," "tâchent de," "cherchent à," "veulent," etc. La plupart des actions offensives relevées dans le tableau précédent en 40 et 50 sont liées à de telles intentions, ce qui change fondamentalement l'image du protestant. Le protestant n'est pas d'abord celui qui agresse la société mais celui qui la menace, celui qui cherche à la perturber. Dans cette image apparaît déjà l'idée d'une perturbation qui n'est pas ressentie comme temporaire. Dans la mesure où le danger ne se situe pas au niveau des actions mais qu'il touche à une volonté de destruction, les édits tout comme les trèves ne pourront être considérés que comme un intermède dans la mise en oeuvre de cette destruction. 
Il est tentant de faire le lien entre les rapports du protestant avec la société catholique du XVIe siècle et ceux du Juif avec la société médiévale. ${ }^{28}$ Le fait qu'ils fassent tous deux partie de minorités religieuses et qu'on ait attribué à l'un et à l'autre le rôle de bouc émissaire masque mal cependant les points de dissemblance entre les deux phénomènes. Toutefois, l'image du Juif dans la société catholique médiévale ayant déjà été étudiée, elle nous servira de point de comparaison. Constituée à partir de documents de la cour criminelle, cette image est généralement négative et elle se manifeste par "des accusations et du mépris à l'égard du Juif en tant que tel." ${ }^{29}$ Les facteurs religieux revêtent ici une importance particulière puisqu'une part considérable des accusations porte sur l'insulte faite à la foi chrétienne. Le réseau des qualifications et des oppositions a montré l'importance accordée à la religion dans la constitution de l'image du protestant. Les autres réseaux ont cependant nuancé largement cette importance en replaçant la religion au sein même du système culturel dont elle ne devient dès lors qu'un élément. L'image du Juif révèle en outre que les chrétiens prêtent aux Juifs "l'intention de menacer leur existence." 30 Cette intention est également prêtée aux protestants et il semble bien qu'on ait là le trait fondamental de la représentation que les Aixois s'en font. Il faudrait pousser plus loin la comparaison entre le Juif médiéval et le protestant du XVIe siècle, à partir de cette menace à l'existence catholique qu'ils partagent. La piste permettrait sans doute d'approfondir nos résultats.

$$
* * *
$$

Les documents ne nous ont pas permis de vérifier avec précision si l'image du protestant reposait sur l'observation d'une culture considérée comme étrangère. Une étude conceptuelle poussée visant à définir les éléments qui créent l'étranger du point de vue culturel manque ici pour considérer une solution au problème posé. Le terme d'étranger qui apparaît dans les délibérations municipales semble d'abord faire appel à une notion juridique assez éloignée de la notion de distance culturelle qui nous occupe ici. ${ }^{31}$ Pourtant, l'étude approfondie de la notion juridique permettrait peut-être d'éclairer un tant soit peu le problème en précisant comment les Aixois protégeaient leur propre existence.

La notion d'étranger, pour autant que je puisse la percevoir, suppose le refus d'intégrer dans son propre système des éléments nouveaux. Deux attitudes peuvent en découler:

1o - l'indifférence qui permet la coexistence et amène à plus ou moins long terme une forme d'acculturation;

2o - la réaction violente qui cherche prétexte de l'agression étrangère pour accentuer ses propres barrières culturelles.

Dans cette optique, il semble que le conseil aixois ait façonné l'image 
des protestants en insistant sur la menace qu'ils constituaient pour le système culturel catholique aixois dont ils sapaient lentement les bases (roi-religion) et qu'ils souhaitaient finalement détruire. Dans ce sens, la notion d'étranger est alors fortement liée à celle de protestant.

Après 1564, le conseil doit compter en son sein le quart de conseillers protestants. La présence des conseillers protestants fait que ceux-ci disparaissent dans le discours des procès-verbaux et l'on perd finalement l'image que les années des troubles avaient constituée. Cette représentation se transforme-t-elle? Il faudra poursuivre la recherche pour s'en assurer mais je serais portée à le croire. L'autre attitude découlant de la perception de l'étranger a bien pu prendre le dessus à la suite d'une coexistence imposée et il ne serait pas étonnant que les deux cultures aient subi, malgré elles, les effets d'une acculturation.

\section{Université Laval}

Notes

1 Notons pour mémoire l'article de M.-H. Froeschlé-Chopart, "Les dévotions populaires d'après les visites pastorales; un exemple, le diocèse de Vence au début du XVIIIe siècle," Revue d'histoire de l'Eglise de France, t, 60, no. 164 (janvier-juin 1974), p. 85-99, et la thèse maintenant classique de Michel Vovelle, Piété baroque et déchristianisation en Provence au XVIIIe siècle. Les attitudes devant la mort d'après les clauses des testaments. Paris, Plon, 1973, 697 pages.

2 Janine Estèbe, Tocsin pour un massacre. La saison des Saint-Barthélemy. Paris, 1968, et Natalie Zemon Davis, "The Rites of Violence: Religious Riot in Sixteenth-Century France," Past and Present, no 59 (May 1973) p. 53-91. Le débat entre ces deux historiennes s'est engagé dans la revue Past and Present no 67 (May 1975), p. 127-135.

3 Denis Richet, "Aspects socio-culturels des conflits religieux à Paris dans la seconde moitié du XVIe siècle," Annales E.S.C., 32e année, no 4 (juillet-août 1977), p. 765.

4 On sait avec quelle habileté Philippe Joutard a étudié l'historiographie portant sur les camisards d'abord, sur la Saint-Barthélemy ensuite. On ne peut plus, après de telles études, utiliser naivement les Histoires du protestantisme sans leur adjoindre une histoire de l'histoire. Philippe Joutard, La légende des camisards. Une sensibilité au passé. [Paris] Gallimard [1977], $439 \mathrm{p}$. (coll. Bibliothèque des Histoires), et Philippe Joutard, Janine Estèbe, Elisabeth Labrousse et Jean Lecuir, La Saint-Barthélemy ou les résonances d'un massacre, Neuchatel, Delachaux et Niestlé [1976], 245 p.

$5 \mathrm{~J}$. Melville Herskovits, Les bases de l'anthropologie culturelle traduit de l'anglais par François Vaudou. Paris, Payot, 1952, p. 61 (Man and his Works).

6 Les problèmes d'acculturation et de transmissions culturelles ont été étudiés par Herskovits mais ils ont été repris dans la perspective historique par Alphonse Dupront, "De l'acculturation." Rapports, T. I Grands thèmes: Comité international des sciences historiques. XIIe Congrès international des sciences historiques, Vienne, 29 août- 5 septembre 1965. Vienne, Verlag Ferdinand Berger \& Söhne, p. 7-36, et par N. Wachtel, "L'acculturation," Faire de l'Histoire, T. I, Nouveaux problèmes [Paris] Gallimard [1974], p. 124-146. Nous citons ici A. Dupront, loc. cit., p. 28.

7 Voir surtout Régine Robin, Histoire et linguistique, Paris, Armand Colin [1973], 306 pages. Je tiens à remercier madame Robin d'avoir bien voulu relire cet article en y apportant ses commentaires. 


\section{4 / Renaissance and Reformation}

8 Le texte ici ne suggérait rien puisque nous ne lui avons pas fait subir au préalable les calculs de fréquence, d'occurrence et de cooccurrence.

9 Nous nous sommes ici inspiré de l'exemple donné par R. Robin, op. cit., p. 140-142, que nous avons simplifié au maximum. Nous avons finalement laissé de côté la catégorie "action sur," peu importante et trop disparate pour être interprétée.

10 Voir précédemment et la note 4.

11 Arch. mun. d'Aix, BB 61 fol. $9 \mathrm{v}^{\circ}$ et $67 \mathrm{v}^{\circ}$.

12 Ibid., BB 61 fol. 31 ss. et fol. $96 \mathrm{v}^{0}$.

13 Ibid., BB 57 fol. 52 ss.

14 Bruno Durand, La vie municipale à Aix en Provence avant 1789. Marseille, Société anonyme du sémaphore de Marseille, 1935, p. 61.

$15 \mathrm{Ph}$. Joutard et al., La Saint-Barthélémy. ..., p. 15.

16 Gustave Lambert, Histoire des guerres de religion en Provence (1530-1598), Nyons, Chantemerle, 1972 (édité d'abord en 1868), t. I, p. 110 ss.

17 Ibid., p. 120 ss.

18 Arch. munc. d'Aix, BB 59 fol. $48 \mathrm{v}^{\circ}$.

19 G. Lambert, op. cit., p. 220 ss.

20 L'obligation faite par l'édit que le conseil soit composé pour le quart de protestants n'explique qu'en partie cette situation.

21 P.J. De Haitze, Histoire de la ville d'Aix capitale de la Provence, Aix, Publication de la Revue Sextienne, Librairie Makaire, 1883, t. 2, p. 387.

22 Arch. dép. des Bouches-du-Rhône, G 146, fol. 21.

23 Nos sources pour l'instant se limitent aux testaments relevés pour l'année 1572, et aux abjurations. La piste la plus rentable semble celle des testaments. Seule une étude systématique de la société aixoise permettrait d'y découvrir la place des protestants de façon assurée. Les meilleurs connaisseurs de la question pour la Provence ne se prononcent d'ailleurs qu'avec grande prudence sur la composition sociologique de la communauté. Ph. Joutard et al., La Saint-Barthélémy. ... , p. 13 et Marc Venard, "Les protestants du Comtat Venaissin au temps des premières guerres de religion," Actes du colloque l'A miral de Coligny et son temps. (Paris, 24-28 octobre 1972) Paris, Société de l'histoire du protestantisme français, 1974, p. 275 à 299 et p. 344-346.

24 Comme le fait D. Richet, loc. cit.

25 M. Venard, loc. cit., p. 346, note qu'en 1560 le mot huguenot apparaît en France, qu'il remplace celui de luthérien et qu'il cèdera ensuite sa place à l'expression "ceux de la nouvelle religion."

26 Il est bien entendu que les protestants, de leur côté, agissaient de même. "Sûrs d'eux-mêmes, les notables protestants entendent rester sur le terrain de la légalité et affirment lutter contre la "rébellion" et la "sédition." En aucun cas, ils ne se veulent des conjurés." D. Richet, loc. cit., p. 769.

27 Les connotations positives relevées ici et plus bas sont dues essentiellement à une intervention du comte de Morsant venu au conseil le 18 octobre 1564 pour faire appliquer l'édit de pacification. Arch. mun. d'Aix, BB 60 fol. $76 \mathrm{v}^{\circ}$ ss.

28 Danièle Iancu-Agou, "Les Juifs de Provence entre l'exclusion et l'expulsion (fin XVe siècle)," Revue des études juives, t. 136, fasc. 3-4 (juil.-déc. 1977), p. 537-543 fait ce rapprochement.

29 Joseph Shatzmiller, Recherches sur la communauté juive de Manosque au Moyen Age, 1241 1329. Préface de Georges Duby, Paris, Mouton, 1973, p. 122.

30 Ibid., p. 131.

31 Par ex. Arch. mun. d'Aix, BB 61 fol. 142, BB 59 fol. 30, etc. 